\title{
Changes in motor innervation and cholinesterase localization induced by botulinum toxin in skeletal muscle of the mouse: differences between fast and slow muscles
}

\author{
L. W. DUCHEN \\ From the Department of Neuropathology, Institute of Psychiatry and the Maudsley Hospital, London, S.E.5 $\frac{\frac{5}{\bar{T}}}{\frac{\sigma}{\infty}}$
}

Botulinum toxin causes paralysis of skeletal muscle by preventing the release of acetylcholine at motor nerve terminals (Burgen, Dickens, and Zatman, 1949). It is known that, when a sub-lethal quantity of toxin is injected directly into muscles of experimental animals, paralysis and atrophy of those muscles develop and persist for a long time (Guyton and MacDonald, 1947; Jirmanova, Sobotkova, Thesleff, and Zelena, 1964; Duchen and Strich, 1967, 1968). A remarkable overgrowth of motor nerve fibres occurs while the muscles are paralysed and atrophied (Duchen and Strich 1968) and changes in the localization of cholinesterase activity develop. After some weeks muscular function recovers, the nerve growth ceases and then regresses, and the motor innervation of the muscle and the distribution of cholinesterase become reorganized. The pattern of innervation and the morphology of subneural apparatuses remain abnormal permanently. Although the histological investigations of Duchen and Strich (1968) showed that abnormalities develop in the innervation and in the morphology of the subneural apparatuses, the exact relationship between the changes in cholinesterase localization and the sprouting of motor nerve fibres was not determined. This paper presents the result of recent histological studies of the effects of botulinum toxin on skeletal muscle of the mouse in which the relationship of the motor nerve growth to the development of abnormalities in cholinesterase and its localization was examined.

In recent experiments it has become apparent that there are marked differences in the rate at which abnormalities develop in soleus and gastrocnemius and these differences can be correlated closely with the histological characteristics of these muscles which allow their muscle fibres to be designated as 'fast' or 'slow'. The differences in the histological changes induced by botulinum toxin in the various muscles are described in this paper.
These observations have been briefly presented in a preliminary communication (Duchen, 1969).

MATERIAL AND METHODS

Adult male albino mice weighing 20 to $30 \mathrm{~g}$ were used in all experiments. They were fed on diet 41B sup-i plemented occasionally with wheat grain and allowed unlimited access to food and water throughout the experimental period. Under light ether anaesthesia, $\bar{O} \mathrm{O} a \hat{\circ}$ single injection of botulinum toxin was made into gastrocnemius muscle of the right leg. The injection, sufficient to cause paralysis of the local muscles, con-T sisted of about $0.005 \mu \mathrm{g}$ of precipitated Type A to $9 \mathrm{~A}^{\mathbb{D}}$ in $0.05 \mathrm{ml}$. gelatin-phosphate buffer $p \mathrm{H} 6.6$. The mige were killed at intervals ranging from two days to 550 months after the injection of toxin. More than 200 animals have been studied. As controls, the muscles $\vec{G} \overrightarrow{\mathrm{fo}}$ normal young and old mice were studied. Other con-o trols included muscles of the left leg of the experimental animals as well as muscles of mice which hado received injections of botulinum toxin previously inactivated by being heated to boiling point.

HISTOLOGICAL METHODS Mice were killed under ether anaesthesia by an incision into the right auricle of the $\Rightarrow$ heart. For the demonstration of cholinesterase the leg⿳亠丷厂 muscles were fixed by perfusion, through the aorta, with cold $\left(4^{\circ} \mathrm{C}\right)$ formol-calcium $(10 \%$ formalin in $1 \%$ calcium acetate). The calf muscles were then dissectedout, and immediately frozen in acetone cooled with solid carbon dioxide. Serial longitudinal frozen sections were cut at $20 \mu$ and stained by the method essentiallyo as described by Koelle and Friedenwald (1949) modified 3 by Lewis (1961) and Henderson (1967). Silver impregnation of nerve fibres after the cholinesterase reactions was carried out by the method of Namba, Nakamura, and Grob (1967). The substrate used was either acetyl-응 or butyrylthiocholine iodide and incubation was carriedt out at room temperature. When enzyme activity alone was demonstrated the $p \mathrm{H}$ of the substrate was adjusted to $5 \cdot 6$, the incubation time was 20 minutes and sections were subsequently dipped in $0.5 \%$ silver nitrate whichN blackens the precipitate of reaction product and renders 
the preparation permanent. The $p \mathrm{H}$ of the substrate was kept down to about $5 \cdot 2$ and the incubation time limited to 10 to 15 minutes when nerve fibres were to be stained after the cholinesterase reaction. Limitation of incubation time at this $\mathrm{pH}$ allowed greater clarity of nerve fibre staining than could be achieved at the higher $p \mathrm{H}$, though less enzyme activity could be demonstrated. Some sections were treated with tetra-isopropylpyrophosphoramide (iso-OMPA) in concentration of $3 \times$ $10^{-6} \mathrm{M}$ for half an hour before, and during, incubation in the substrate in order to inhibit nonspecific cholinesterase activity.

Some mice were fixed by perfusion with formol alcohol (10\% formalin in $60 \%$ alcohol) and, after decalcification, blocks of tissue were embedded in gelatin and nerve fibres impregnated with silver by Schofield's method in serial thick $(60 \mu)$ frozen sections. Other animals were fixed by perfusion with formolacetic-methanol $(10 \%$ formaldehyde, $10 \%$ glacial acetic acid, $80 \%$ absolute methanol). After decalcification, multiple transverse blocks of spinal cord and limbs were embedded in paraffin wax and sections stained by routine neurohistological methods.

The distribution in the muscles of the leg of the two enzymes succinate dehydrogenase and phosphorylase was studied in normal mice. Cryostat sections were cut of fresh tissue frozen in iso-pentane cooled with liquid nitrogen. Succinate dehydrogenase was demonstrated by the method of Nachlas et al. using Nitro-BT as described by Pearse (1960). Phosphorylase was demonstrated by the method of Lake (unpublished).

After differences between soleus and gastrocnemius had been observed it was considered useful to examine the distribution of dye injected in the same way as botulinum toxin. Pontamine sky blue was used and $0.05 \mathrm{ml}$. of a $20 \%$ solution of the dye in gelatin-phosphate buffer was injected into the right gastrocnemius of 10 mice which were killed at intervals ranging from 15 minutes to 20 hours after the injection. Dye became rapidly diffused throughout the leg muscles, spread along fascial planes within 30 minutes, and began to spread over the thigh muscles and over lower spinal muscles within two hours. At 20 hours there was generalized blue staining of the tissues which was most intense over the surface of the gastrocnemius and distal half of the hamstring muscles. There was no pooling of the dye around soleus.

\section{RESULTS}

CLINICAL Paralysis of the muscles of the right hind leg developed within about 24 hours after the injection of the toxin. There were also transient signs of generalized toxicity which consisted of muscular weakness and respiratory difficulty with costal recession. The right leg was obviously paralysed for three to four weeks and atrophy of the limb muscles became apparent. During the first few weeks after the injection of toxin, the mouse was unable to plantarflex the foot, which could not be used for gripping the bars of the cage during climbing activity. Recovery of the use of the foot and the reappearance of plantar flexion began at about the fifth week and by the tenth week the mouse could use the leg and foot well, though not normally. Even the longest surviving animals tended not to use the right hind leg and foot during climbing activity and sometimes the right foot would be used clumsily or miss its grip, a most unusual finding in a normal mouse.

HISTOLOGICAL In the normal mouse the innervation of extrafusal skeletal muscle conforms to the general pattern of innervation seen in mammals (Coèrs and Woolf, 1959). Nerve trunks enter the muscle at the neurovascular hilum and much axonal branching takes place in the nerve trunks and bundles as they spread through the muscle. Near the middle of the muscle each nerve bundle breaks up into a spray of single myelinated axons each of which proceeds for a short distance before terminating in the motor end-plate of a single extrafusal muscle fibre. The delicate unmyelinated nerve fibres of the terminal arborization lie in gutters of the sarcolemmal membrane in close apposition to the 'subneural apparatus' formed by infoldings of the sarcolemmal membrane (Couteaux, 1958) in which cholinesterase activity is particularly concentrated. The shape and size of the subneural apparatus varies with the plane of the section. When the whole end-plate is included in the section the appearance of the subneural apparatus is often, particularly in gastrocnemius, that of a ring-like structure with internal branches (Fig. 1). Branches are not usually seen pointing outwards from the periphery. Incubation in acetylthiocholine iodide substrate at $p \mathrm{H} 5.6$ demonstrates more reaction product than at $p \mathrm{H} 5 \cdot 2$. In the presence of isoOMPA the intensity of the reaction in subneural apparatuses is slightly reduced. Incubation in butyrylthiocholine substrate shows nonspecific cholinesterase activity which is inhibited by iso-OMPA. When the incubation is restricted to 10 to 15 minutes no reaction product is seen in the myelinated nerve fibres themselves, but this is seen at $p \mathrm{H} 5.6$ incubated for 30 minutes or more.

The important points about the normal innervation of extrafusal muscle fibres in the mouse relevant to the findings in this work are (1) each muscle fibre is innervated by a single preterminal myelinated axon which usually does not branch after leaving the nerve bundle; (2) each motor nerve fibre terminates within a motor end-plate-that is, there are no motor nerve fibres which end elsewhere than in close relationship to a subneural apparatus with its cholinesterase activity; (3) no motor 


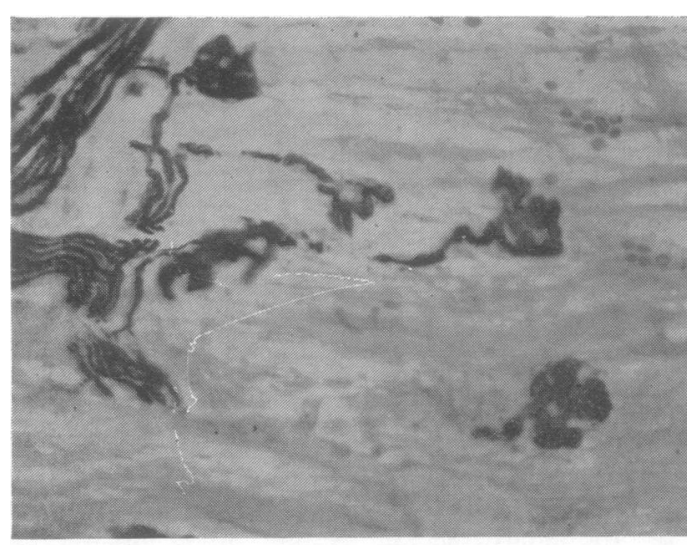

(a)

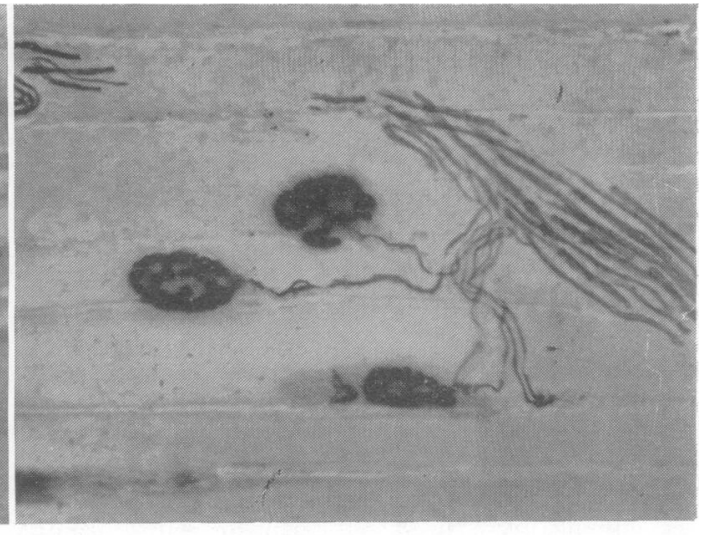

(b)

FIG. 1. Motor nerve terminals in soleus (a) and gastrocnemius (b) of a normal mouse. Each preterminal axon innervates one motor end-plate. The cholinesterase is localized to the subneural apparatuses which appear as rings with branching gutters in (b) and rather simpler stubby gutters in (a). (Cholinesterase and silver impregnation by the method of Namba et al. (1967). Acetylthiocholine substrate $\mathrm{pH} 5 \cdot 2$, incubated 15 minutes, $\times 250$.

nerve fibre extends beyond the end-plate regionthat is, there are normally no 'ultraterminal' nerve fibres.

In the muscles of normal mice histological differences between soleus and gastrocnemius were observed in transverse sections of paraffin embedded tissues, in cholinesterase preparations and in the distribution of muscle enzymes. In transverse sections the muscle fibres of soleus tend to be smaller and more uniform than the fibres of the superficial part of gastrocnemius (Fig. 2). The subneural apparatuses of soleus consist of short, rather simple gutters, whereas in the superficial part of gastrocnemius the gutters typically form an oval or ring with complex internal branching. Soleus muscle fibres are uniformly rich in succinate dehydrogenase (Fig. 3a) whereas in the superficial parts of gastrocnemius most fibres give a weak reaction for this enzyme (Fig. 3b). On the other hand, most of the peripheral muscle fibres in gastrocnemius give a strong staining reaction for phosphorylase, while the fibres of soleus are uniformly weakly stained. In the deeper parts of gastrocnemius the appearance of muscle fibres and subneural apparatuses were like those of soleus.

After the injection of botulinum toxin changes were localized mainly to the calf and hamstring muscles. Atrophy of muscle fibres developed after the injection of toxin and by the end of the first week was more severe in soleus than in gastrocnemius. Impregnation of nerve fibres by silver methods showed that preterminal axons and terminal arborizations were intact. No necrosis of these nerve fibres was seen. In cholinesterase preparations the appearance of the subneural apparatuses pas normal during the first week after the onset $\overrightarrow{0} 0 \hat{6}$ paralysis. There was no evidence of a weaken $\overrightarrow{\mathrm{g}}$ of the staining reaction for cholinesterase activity The first abnormality of nerve fibres was seen $i \frac{8}{8}$ soleus by the sixth or seventh day after the injector of toxin. Sprouting from most, if not all, the mato nerve terminals was seen in soleus (Fig. 4). Thêse nerve sprouts were extremely thin and delicate branched irregularly. Nerve sprouts did not arise from the preterminal myelinated axons. Cholino esterase reaction product was rather diffusely spreac along these sprouting fibres and looked very difo ferent from its orderly concentration in gutters in the normal subneural apparatus. The origina subneural apparatuses could still be easily identified during the early stages of nerve sprouting. Incubatio in butyrylthiocholine iodide substrate showed marked activity along the nerve sprouts (Fig. 5a). Incubation in acetylthiocholine iodide in the presence of iso-OMPA largely abolished the enzyme activity along the nerve sprouts while the enzyme activity in the original subneural apparatuse was well preserved (Fig. 5b). Thus, it was evidenis that most of the cholinesterase along the new grown nerve fibres was nonspecific (or pseudod in type. At the time that the growth of motor nerve fibres was first seen in soleus, the histological aps pearance of gastrocnemius varied in different parts. of the muscle. In the superficial parts of gastrog cnemius the muscle fibres showed some atrophyo but motor nerve fibres and the subneural apparatusess 


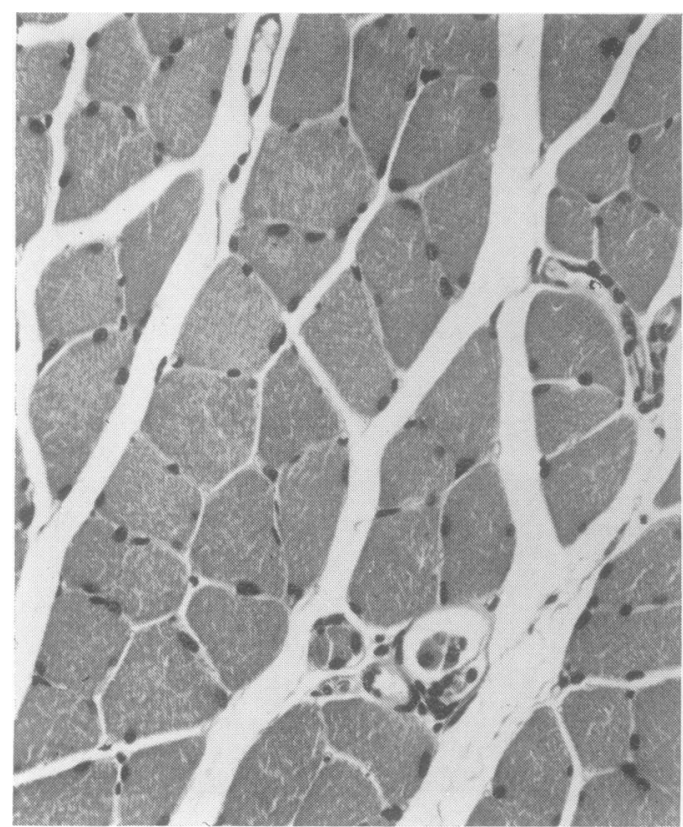

(a)

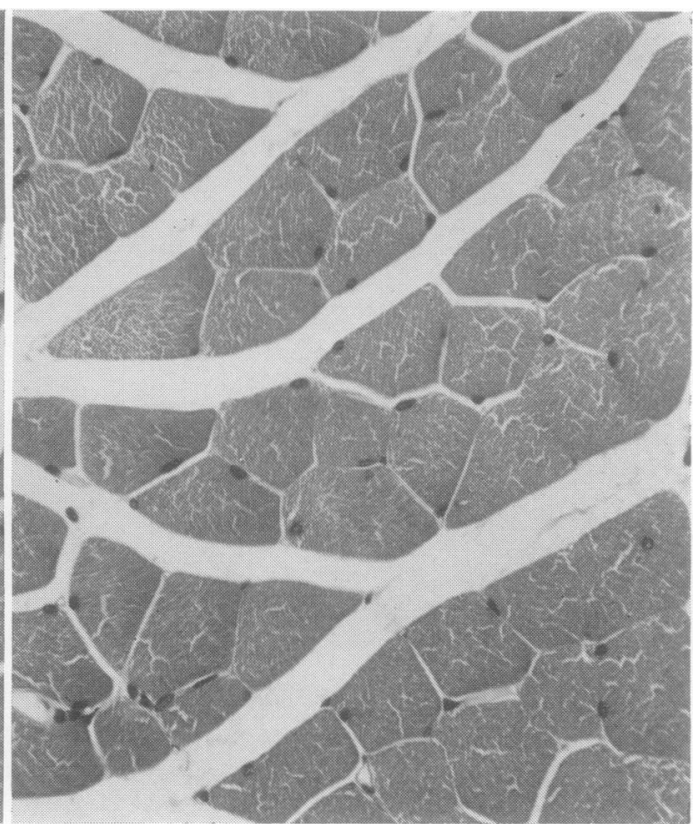

(b)

FIG. 2. Transverse paraffin sections of soleus (a) and of the superficial part of gastrocnemius (b) of the same leg of a normal mouse. All subsequent illustrations show similar regions of soleus and gastrocnemius. $H$ and $E, \times 250$.

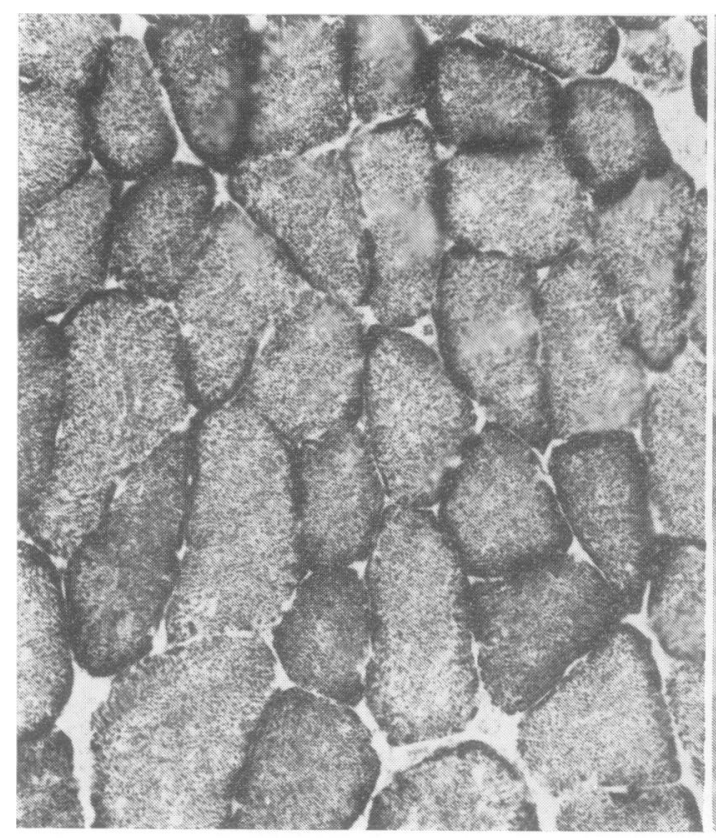

(a)

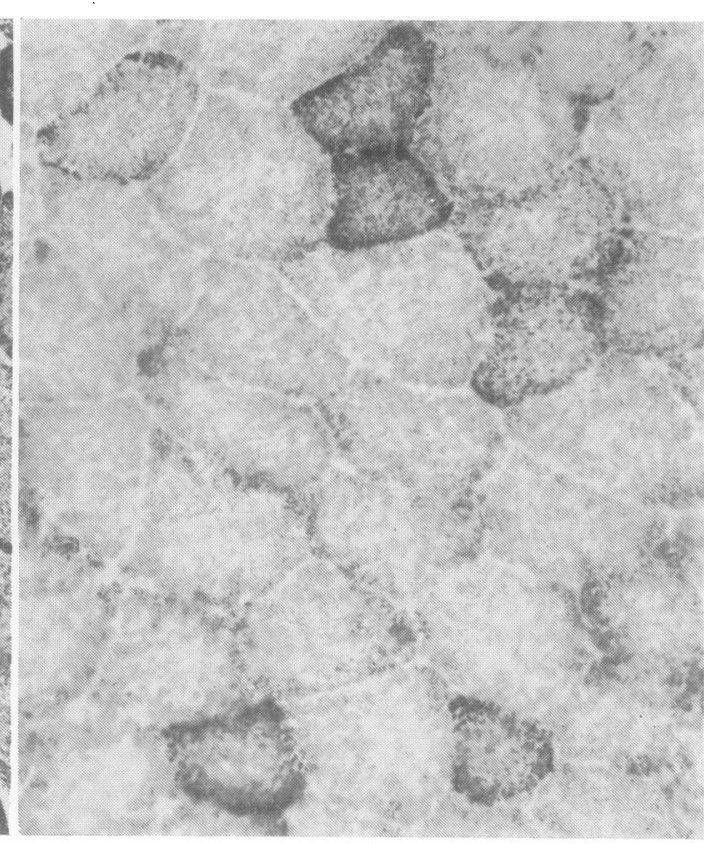

(b)

FIG. 3. Transverse frozen sections of soleus (a) and superficial gastrocnemius (b) stained to demonstrate succinate dehydrogenase. Most of the muscle fibres of soleus but only a few in this region of gastrocnemius give a strong reaction. Succinate dehydrogenase, $\times 250$. 


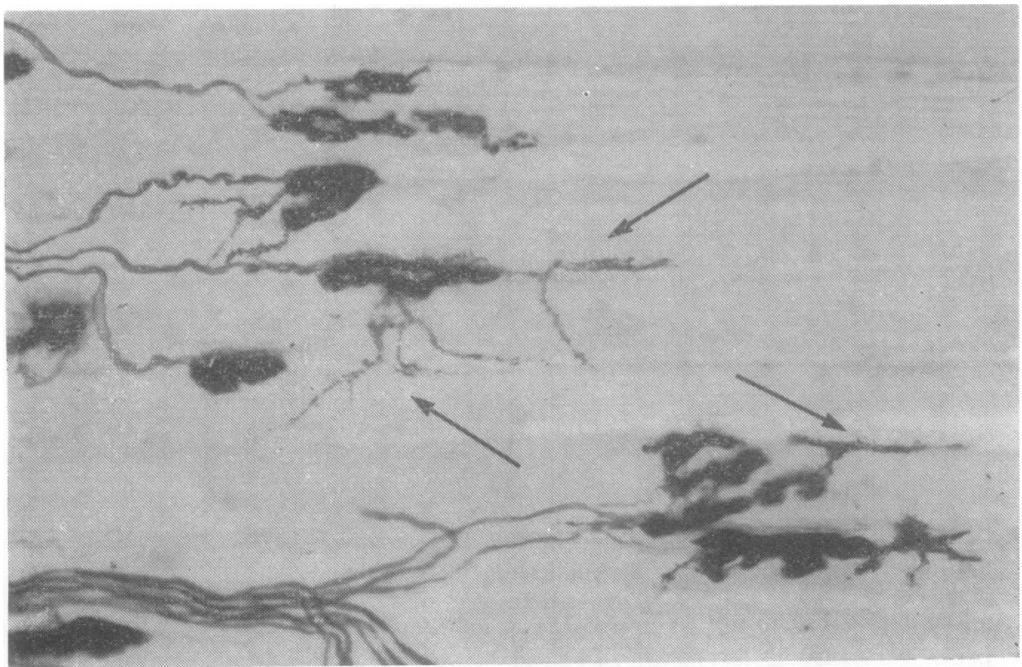

FIG. 4. Motor nerve terminals in soleus one week after the injection of toxin. Delicate branching nerve sprouts (arrows to some) arise from most of the end-plates.

Cholinesterase reaction product $\bar{C}$ is irregularly scattered along the nerve sprouts. Method as in Fig. 1, × 250 .

FIG. 5. Sections of soleus

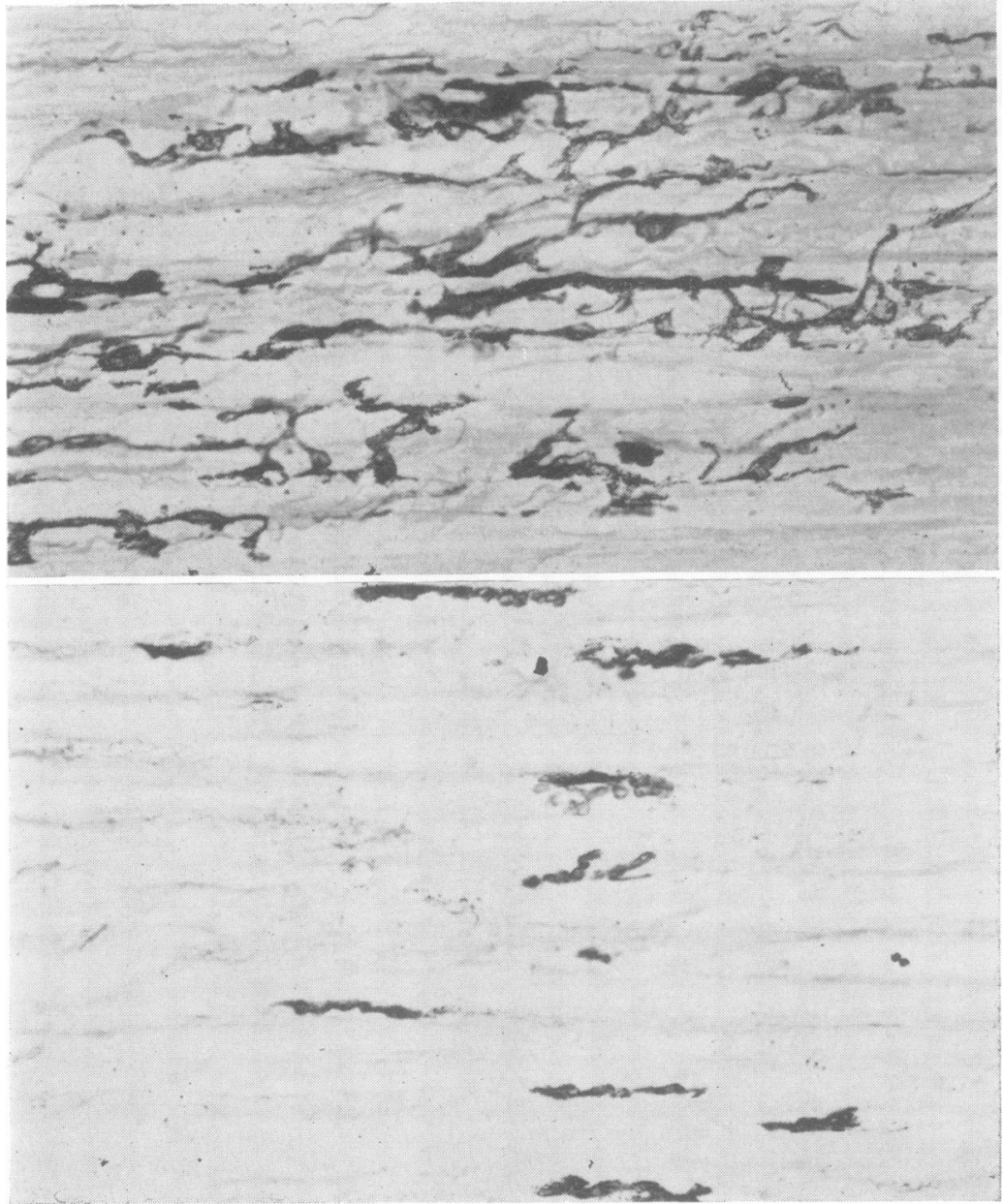
16 days after the injection of toxin. Adjacent sections were incubated in butyrylthiocholine (a), acetylthiocholine with iso-OMPA (b) Pseudo-cholinesterase is localized along the newlyformed ramifying nerve sprouts while true (acetyl) cholinesterase is confined to the gutters of the origina subneural apparatuses. $\times 250$.

(a)

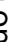
$\overrightarrow{+}$

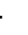


looked normal. The localization of cholinesterase was seen, as in the normal, in well ordered branching gutters and the intensity of the enzyme reaction was normal. In the deeper parts of gastrocnemius nerve sprouting from many of the terminals was seen, while other nerve terminals were normal in appearance. This varied picture of sprouting from some nerve terminals but not from others was also seen in plantaris.

By the second to third week after the injection of toxin the histological differences between soleus and gastrocnemius were striking. Nerve growth was now very marked in soleus with abundant new nerve sprouts growing from almost every motor nerve terminal and spreading between the muscle fibres both longitudinally and transversely. In the deeper parts of gastrocnemius and in plantaris the nerve sprouting was seen at some of the terminals. In the superficial parts of gastrocnemius the nerve terminals were somewhat crowded together because of the atrophy of muscle fibres which was now well marked. The subneural apparatuses were oval or oblong instead of rounded but the enzyme activity was unchanged in its intensity and no nerve sprouting was present yet. Necrosis of scattered single muscle fibres was also seen in gastrocnemius. These necrotic fibres were seen from the second week to the sixth week after the injection of toxin. Muscle fibres in both soleus and gastrocnemius were very atrophied by the end of the second week (Fig. 6). After the second week the atrophy of muscle fibres of the superficial parts of gastrocnemius became very marked and by the third to fourth week was much more severe than in soleus (Fig. 7). The changes in soleus, whether of nerve growth or of muscle fibre atrophy, always tended to be fairly uniform throughout the muscle, whereas in gastrocnemius the variability in different parts of the muscle was characteristic. The atrophy of muscle fibres was always more severe in the periphery of gastrocnemius than in the deeper parts of the muscle. A constant feature seen in the peripheral part of gastrocnemius, however, was that some fibres-either singly or in groups-remained much larger and rounder than the others.

Between the third and fourth weeks the motor nerve growth in soleus was advanced and the pattern formed by nerve fibres very complex indeed. Cholinesterase was distributed in an abnormal fashion with the enzyme localized in the form of branching lines or diffuse patches, following the arrangement of the nerve fibres. At the third week the motor nerve growth and cholinesterase distribution in the deeper parts of gastrocnemius resembled those in soleus, but in the superficial parts of gastrocnemius the nerve growth was not yet far

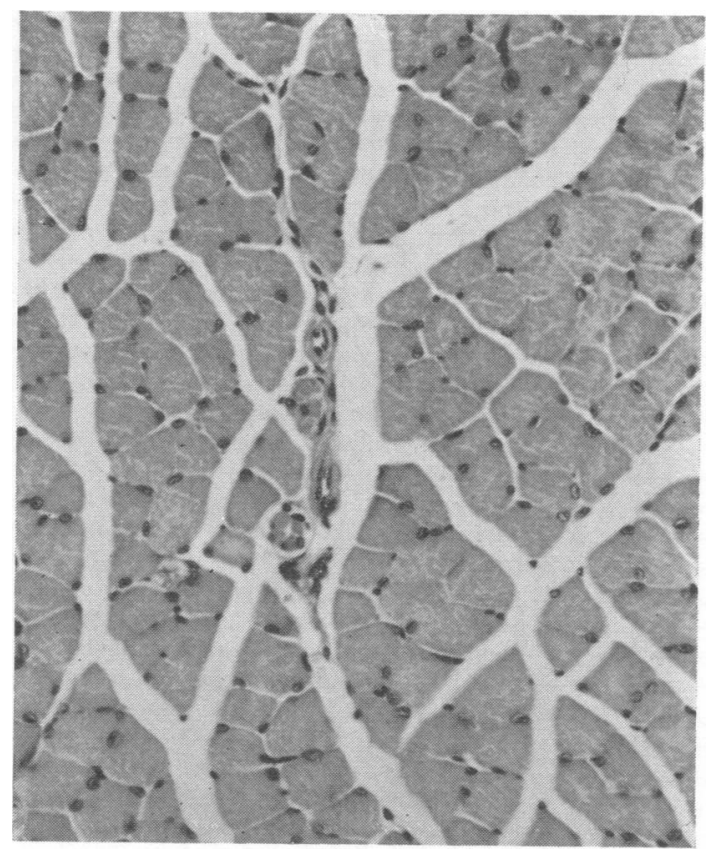

FIG. 6. Transverse section of soleus at two weeks. There is well-marked atrophy of muscle fibres. $H$ and $E, \times 250$.

advanced and many normal-looking subneural apparatuses could still be seen. From this time onwards, however, the motor nerve growth in gastrocnemius proceeded rapidly, eventually reaching the same degree of complexity as in soleus. The nerve growth was always most extensive in relationship to the most severely atrophied muscle fibres. In these areas where nerve growth was most exuberant, the localization of cholinesterase became most abnormal (Fig. 8). Diffuse reaction product lay in close relationship to the nerve sprouts and it became difficult to distinguish the original subneural apparatuses with enzyme activity organized in orderly guttering. Incubation of sections in isoOMPA showed that much of the diffuse cholinesterase along the nerve sprouts was inhibited but the enzyme localized in organized guttering was still demonstrable.

Recovery of muscle fibre size occurred at different times in the various muscles beginning in soleus by the fourth week (Fig. 7). Recovery in the size of the muscle fibres in the superficial parts of gastrocnemius began only after about the sixth to eighth week (Fig. 9). As muscle fibres began to increase in size there was no further nerve growth and the cholinesterase activity became progressively more localized into new recognizable sub- 


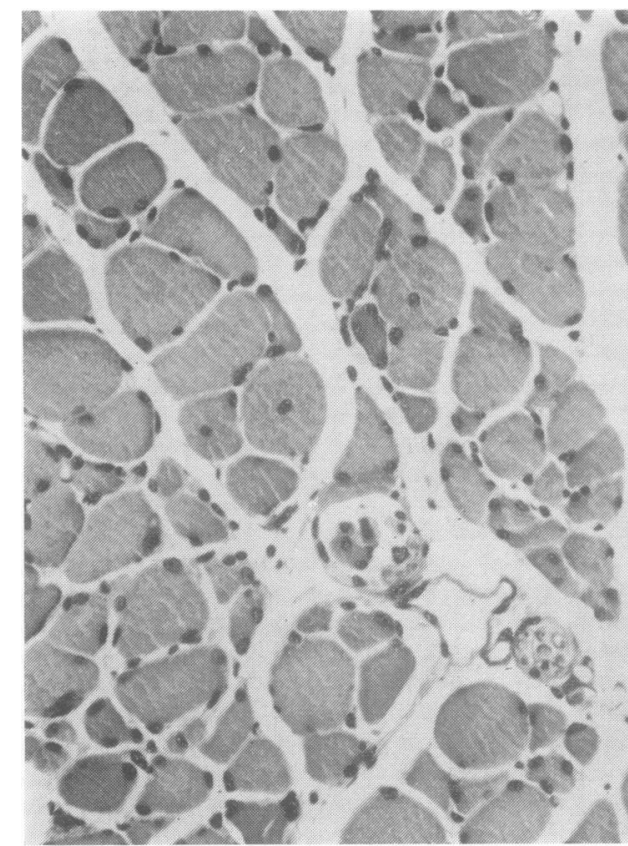

(a)

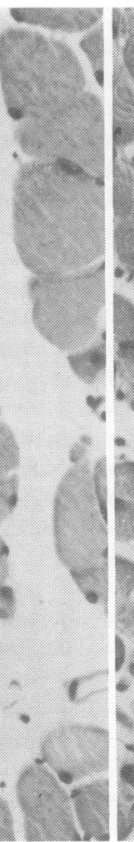

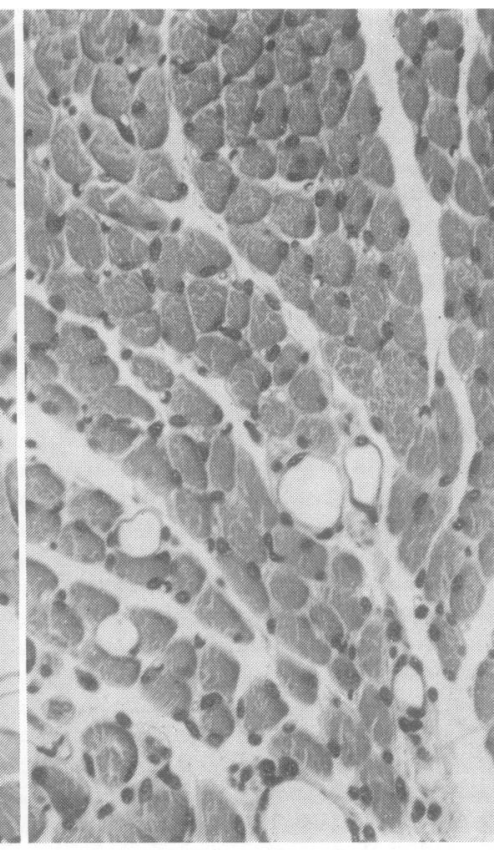

(b)

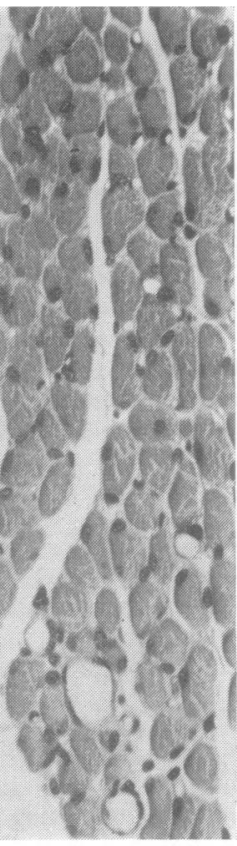

覀

FIG. 7. Transverse sections of soleus (a) and gastrocnemius (b) at four weeks. In soleus some muscle fibres are still atrophied but many are larger than were seen at two weeks. The fibres of gastrocnemius are more severely atrophied than before. $H$ and $E, \times 250$.

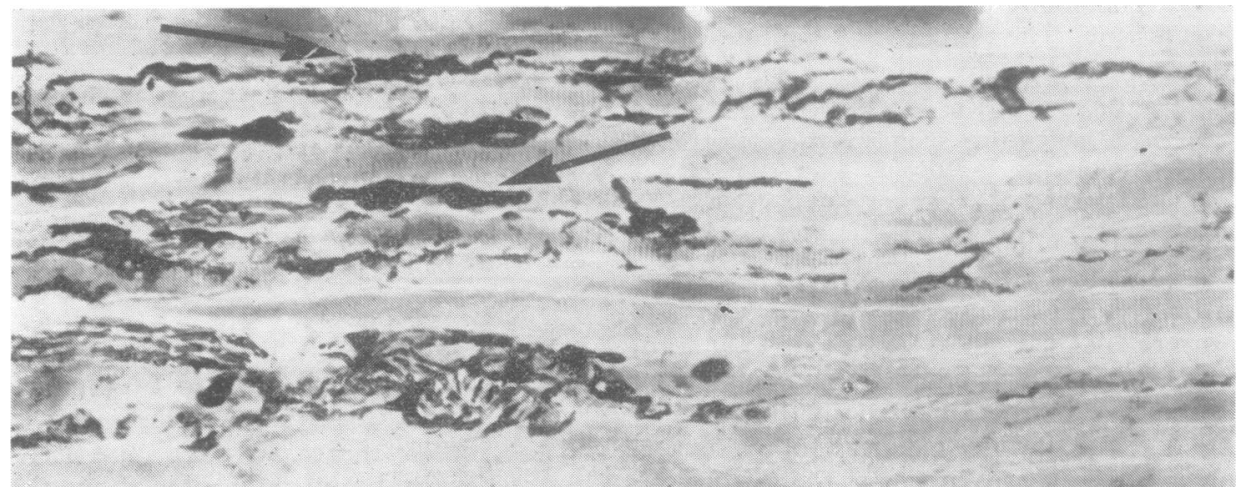

FIG. 8. Gastrocnemius five weeks after the injection of toxin shows severely atrophied muscle fibres with thin branching nerve fibres extending along them. Cholinesterase activity is diffusely spread along these nerve fibres, but some of the original end-plates can still be identified (arrows). Method as in Fig. 1 , $\times 250$. 


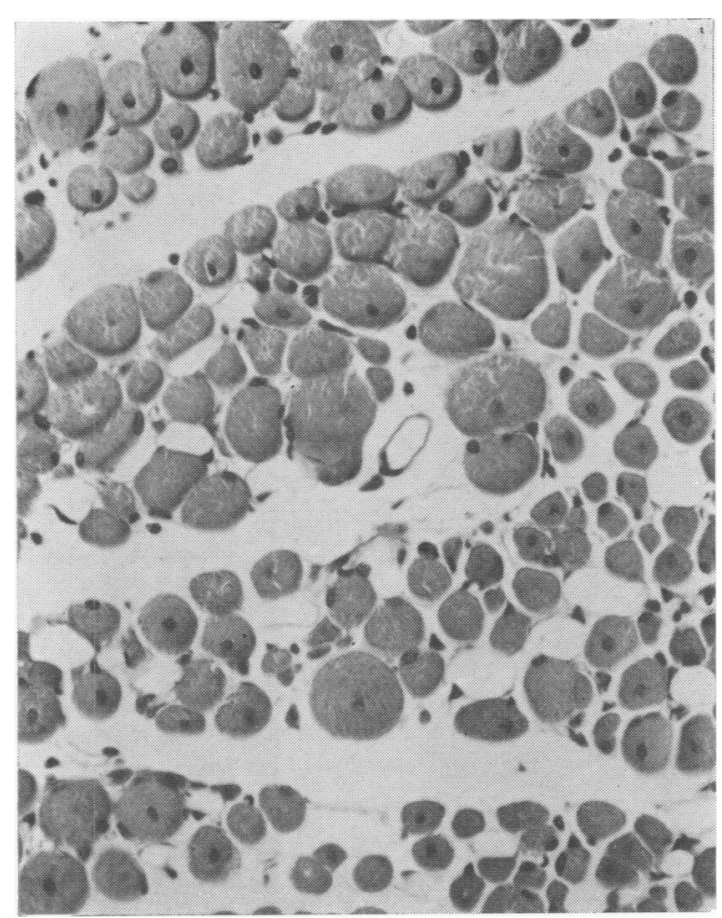

FIG. 9. Transverse section of gastrocnemius at six weeks. Many muscle fibres are larger than at four weeks but others are still atrophied. Centrally placed nuclei are seen in many of the fibres. $H$ and $E, \times 250$.

neural apparatuses. The new subneural apparatuses consisted of localized aggregations of spots, rings or lines (Figs. 10 and 11) and gave a strong reaction for true (acetyl) cholinesterase and a weak pseudocholinesterase reaction. The threads and lines of pseudocholinesterase were no longer seen.
With the cessation of nerve growth, the increasing diameter of muscle fibres and the formation of new subneural apparatuses with gutters, degeneration of some nerve fibres and end-plates was seen. Occasional nerve fibres were irregular in diameter, appearing lumpy or fragmented, and other thin tenuous nerve fibres ended in end-plates where there were only irregular fragments or granules of enzyme reaction product. Degenerative changes of this sort were less frequent with longer survival times. Muscle fibres of soleus were about normal in size by the eighth week. Atrophied fibres persisted in gastrocnemius for up to about six months after the injection of botulinum toxin when they were found either singly or even in small groups (Fig. 12). Central nuclei were seen in many muscle fibres, both large and small, of gastrocnemius, but were found in very few muscle fibres of soleus. Some of the muscle fibres in gastrocnemius were much larger (Fig. 12) than normal. In the animals surviving more than a year there was more than usual variability in size of muscle fibres in gastrocnemius, many with central nuclei, but no severely atrophied fibres were seen at that stage.

In both soleus and gastrocnemius there were similar abnormalities in the pattern of motor innervation and in the morphology of the subneural apparatuses in all the animals which survived for a long time. Some branches of motor nerve fibres appeared to end blindly - that is, not in a motor end-plate (Fig. 13). In many instances it was certain that these were terminals of nerve fibres and not fibres cut off by the plane of the section since they were very thin indeed and it could be determined by focusing at different levels that they ended within the section. Thin nerve fibres were often seen extending beyond an end-plate-that is, these were persisting ultraterminal nerve fibres, which

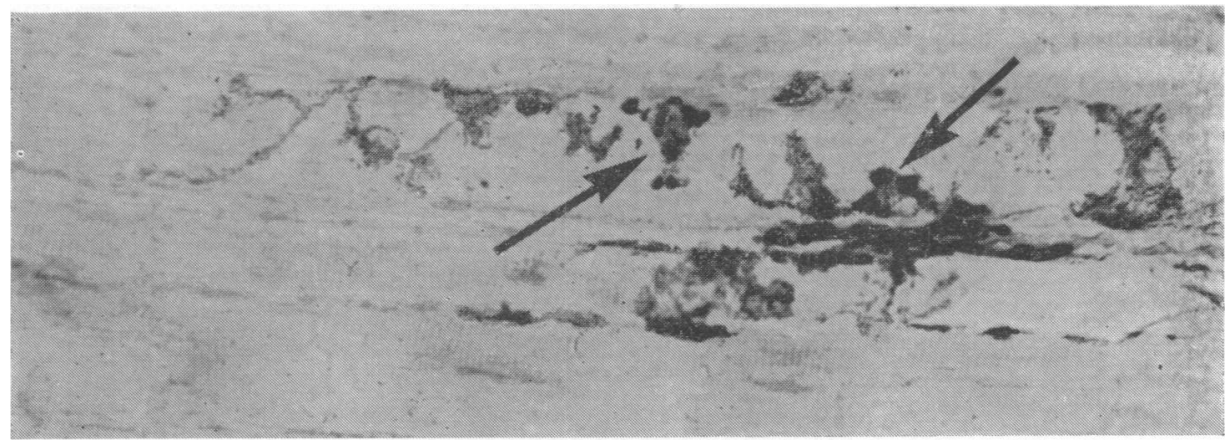

FIG. 10. Soleus at five weeks shows new subneural apparatuses (arrows) consisting of small spots or rings in which cholinesterase is localized. Some of the nerve sprouts still show a little diffuse cholinesterase activity. Method as in Fig. 1, × 250. 


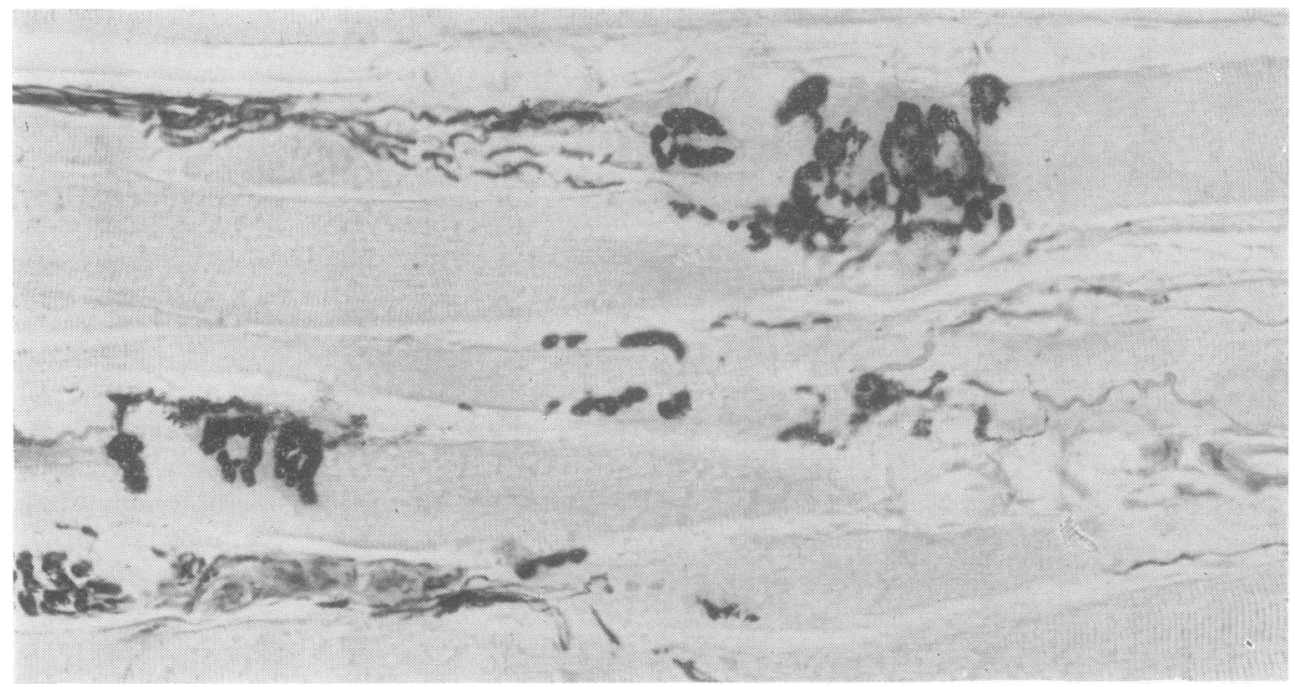

FIG. 11. Gastrocnemius at 12 weeks after the injection of toxin shows new though abnormal-looking subneural apparatusos with cholinesterase localized in gutters. This stage of recovery in gastrocnemius develops several weeks later than in soleus. Method as in Fig. $1, \times 250$.

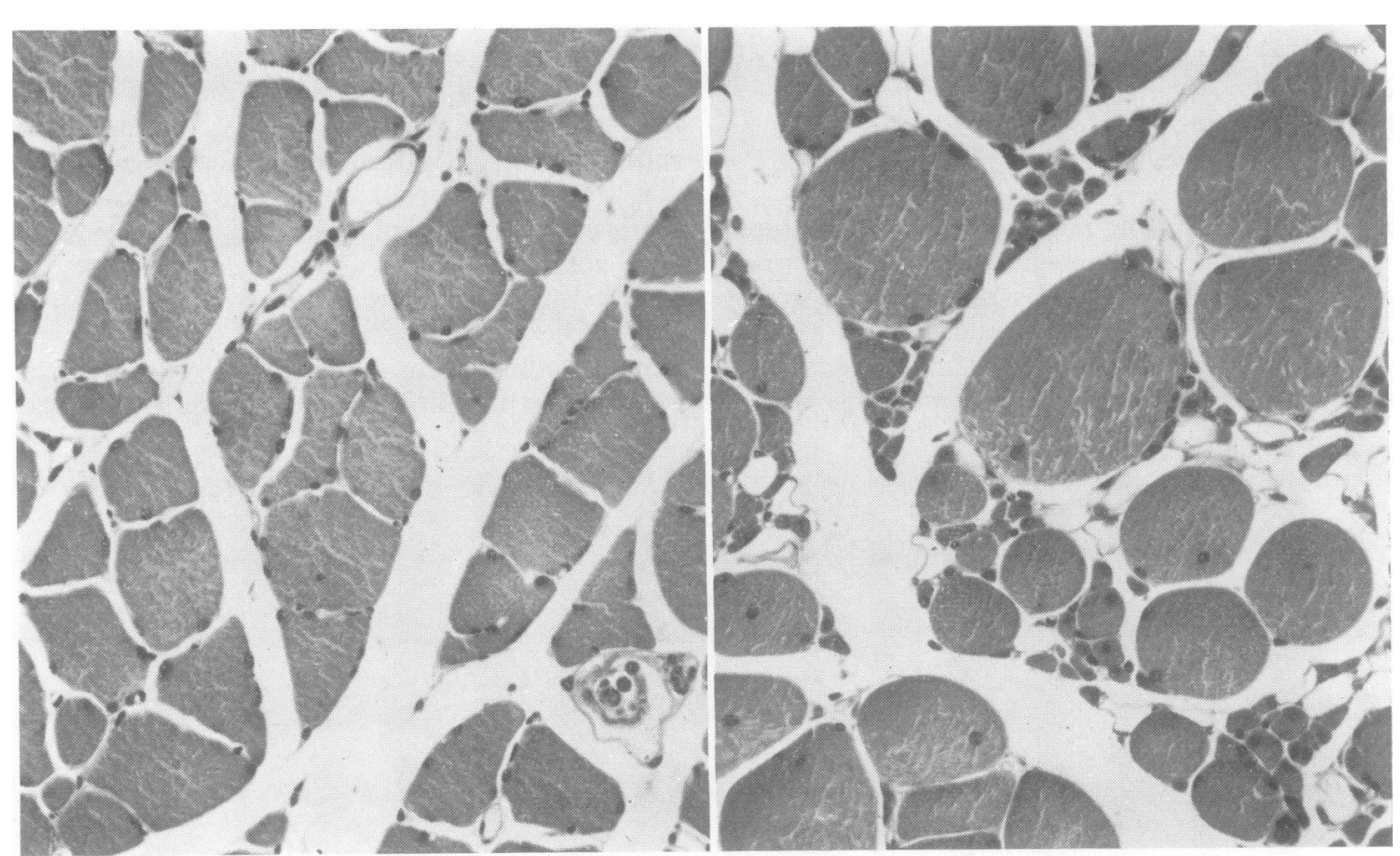

(a)

(b)

FIG. 12. Transverse sections of soleus (a) and gastrocnemius (b) at six months after the injection of toxin. Soleus contains a few atrophied fibres but the appearance is relatively normal. Many muscle fibres in gastrocnemius are still extremely atrophied while others are larger than is seen in the normal. Many fibres have central nuclei. $H$ and $E, \times 250$. 


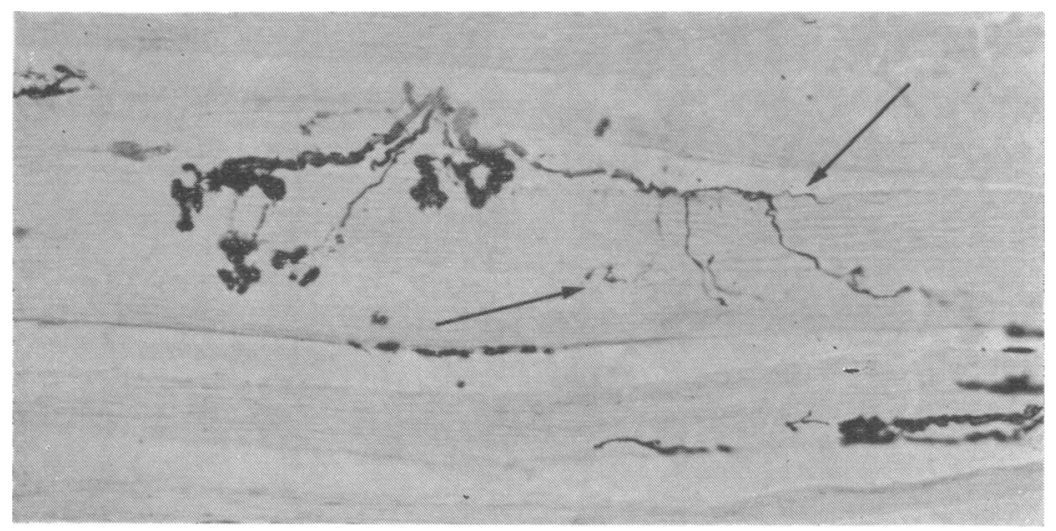

FIG. 13. Gastrocnemius at four months. Some nerve fibres innervate end-plates with strong cholinesterase activity while other thin nerve fibres seem to end 'blindly' (arrows). Method as in Fig. $1, \times 250$.

usually did not appear to innervate another endplate.

In all long-surviving animals the zone of innervation was widened and less well-defined than normal. Preterminal axons were frequently much longer than normal and branching of preterminal axons was seen in all the muscles examined. Branches of some axons innervated several end-plates on different muscle fibres, while in other places several branches seemed to end on the same muscle fibre. The shape and size of subneural apparatuses varied markedly in all affected muscles (Fig. 14). Many were larger and more complex than is ever seen in muscles of normal mice of the same age. It was not possible to distinguish the usual differences in the appearance of the subneural apparatuses in different muscles.

\section{DISCUSSION}

In previous experiments (Duchen and Strich, 1968) it was found that an injection of a sub-lethal quantity of botulinum toxin directly into skeletal muscle in the mouse caused local paralysis and atrophy which persisted for several weeks. During this time there was no degeneration of nerve fibres or of motor nerve endings. Silver impregnations showed that marked sprouting of motor nerve fibres took place and the nerve fibre overgrowth was accompanied by elongation of the sites of cholinesterase activity. As muscle function recovered, nerve fibre overgrowth receded but permanent abnormalities were found in the pattern of innervation and in the morphology of the subneural apparatuses. The present findings extend the observations made by Duchen and Strich (1968), since motor nerve fibres and cholinesterase activity are now demonstrated in the same histological preparations and a clearer understanding of their relationship can be achieved.
It is generally accepted that cholinesterase is present in relation to the junctional folds of the subneural apparatus in greater concentration than elsewhere in nerve or muscle fibres. The final histological picture obtained in any preparation demonstrating cholinesterase activity depends on a number of factors, including the use of fixation and its duration, the $p \mathrm{H}$ of the substrate and the time allowed for incubation, the chemical identity of the substrate, and the use of inhibitors. Acetylcholinesterase is identifiable by its hydıolyzing action on the substrate acetylthiocholine iodide, such activity not being inhibited by the presence of iso-OMPA. Pseudocholinesterase activity is inhibited by iso-OMPA and is also revealed by the hydrolysis of butyrylthiocholine iodide. Denz (1953) showed that, though acetylcholinesterase formed the major component of enzyme at the motor end-plate, pseudocholinesterase was also present. Couteaux (1958) pointed out that several different methods should be used in the histological assessment of cholinesterase activity. Davis and Koelle (1967) demonstrated acetylcholinesterase in association with the pre- and post-junctional membranes of the junctional folds and pseudocholinesterase in relation to the teloglial Schwann cell.

Botulinum toxin blocks neuromuscular transmission within about 24 hours of its administration and the muscle fibres are paralysed from this time onwards for several weeks. During this period of neuromuscular block the release of acetylcholine is prevented (Burgen et al., 1949) yet the intensity of the cholinesterase staining reaction is not altered, and the morphology of the subneural apparatuses becomes abnormal only when nerve sprouting begins, which may be several weeks later. This suggests that the cholinesterase activity of the subneural apparatus is maintained or 


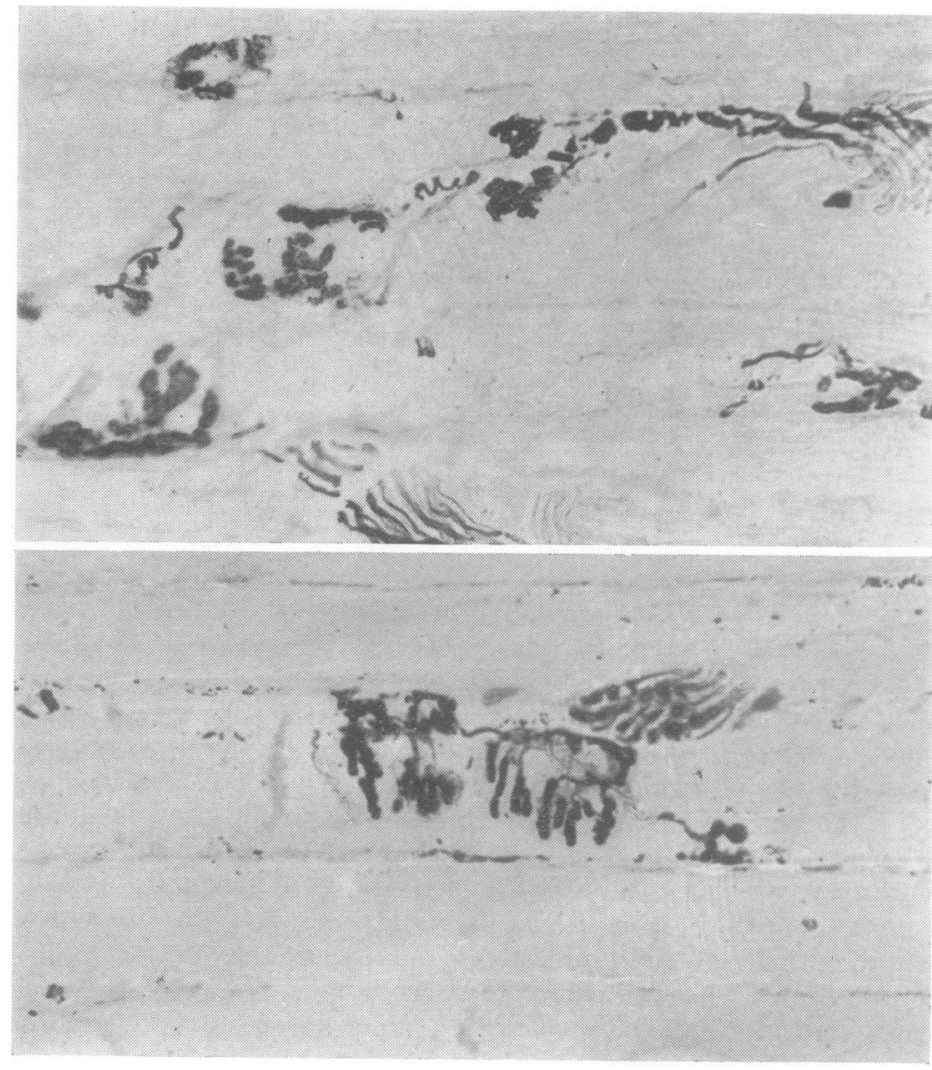

FIG. 14. Subneural apparatuses in soleus (a) and gastrocnemius (b) are very abnormal in appearance at 10 months after the injection of toxin.

They consist of aggregations of subunits in which cholinesterase activity is well localized in gutters. The differences between gastrocnemius and soleus as seen in the normal (compare with Fig. 1) can no longer be clearly seen. Method as in Fig. $1, \times 250$.

influenced by the intact nerve fibre independently of the release of acetylcholine.

The characteristics of the cholinesterase demonstrated in relation to the newly formed sprouting nerves differ in several important respects from the enzymes which are normally found at the end-plate. The crystals and granules of the reaction product lie along the new nerve sprouts in a rather diffuse fashion with irregular aggregations in places, differing greatly from the well-organized gutters and folds of the normal subneural apparatus. The enzyme reaction obtained with butyrylthiocholine as the substrate is strong, whereas in the normal subneural apparatus it is weak. With acetylthiocholine as substrate the reaction along the sprouting nerves is largely abolished by iso-OMPA. The enzyme of the new nerve sprouts is thus mainly pseudocholinesterase and may be related to the Schwann cells which proliferate and accompany the formation of new axoplasm. It is not clear why the newly grown nerve sprouts and Schwann cells should show such strong non-specific cholinesterase activity compared with the normal subneural apparatus and with myelinated preterminal axons or other unmyelinated cholinergic nerve fibres; which show little or no enzyme reaction product under comparable incubation conditions. No known function has been attributed to pseudocholinesterase in experimental animals, though in rare human cases hereditary disorders of pseudocholinesterase are known to cause adverse reactions to some neuromuscular blocking agents (Lehmann, Silk, and Liddell, 1961).

The motor nerve sprouting occurred while the muscle fibres were paralysed and becoming progressively more atrophied. When muscle fibres began to increase in diameter again, there was a cessation of motor nerve growth, new subneural apparatuses were formed, apparently randomly, and there was a progressive diappearance of pseudocholinesterase located along the nerve fibres.

The use of the histological method allowing the demonstration of cholinesterase and nerve fibres in the same preparation has clearly shown that, in the later stages of the experiment when recovery of muscular function and the reorganization of its innervation are taking place, there are several striking abnormalities which were not identified 
in the earlier work. Many branches of nerve fibres ended 'blindly'. Some of these branches were clearly branches of motor nerve fibres since other branches of the same axons terminated in end-plates. It had to be established that these nerve fibres were not cut off by the plane of the section, but their delicacy and the fact that tissue was present in planes of focus both above and below them was convincing evidence of their truly terminal nature. Such blind endings were fewer with longer survival. They were never seen in muscles of normal animals.

In long-surviving animals preterminal myelinated axons were branched and innervated more than one muscle fibre, unlike the normal pattern of innervation in which one preterminal axon usually innervates only one end-plate. There are several possibilities to account for the development of preterminal axonal branching. It could be considered that some sprouts arise from preterminal axons as described in partial experimental surgical denervation (Edds, 1950; Hoffman, 1950). Preterminal sprouting was never seen during the early stages of nerve growth in my experiments, though later the pattern of innervation became so complex and the nerve fibres so numerous that it would be difficult, if not impossible, to exclude such a type of nerve sprouting. It is clear, however, that the characteristic pathological change induced by botulinum toxin is nerve sprouting from the terminals within the end-plate region. In some musclesfor example, soleus-terminal sprouting is found at almost every motor nerve ending within the first week of the experiment. It is most likely, therefore, that the branched preterminal axons found in the late stages of the experiment are the end-result of the remodelling of the motor innervation of muscle during recovery from the effects of the toxin. Some of the end-plates, where sprouting had begun, probably become non-functioning and disappear after new end-plates are formed by what were at one time ultraterminal fibres (Fig. 15).

These histological investigations have shown that there are marked differences between the various muscles in the leg of the mouse in the changes which develop after the local injection of botulinum toxin. Some of these differences are essentially in the rate at which histological changes occur. In soleus and in the deeper parts of gastrocnemius, as well as as in some fibres of plantaris, the growth of motor nerve fibres begins within six or seven days of the injection of toxin, while in the superficial parts of gastrocnemius the motor nerve growth can be seen only after three or four weeks. The actual histological appearances of the motor nerve growth, such as the origin of the nerve sprouts from motor nerve terminals, their spread along muscle fibres

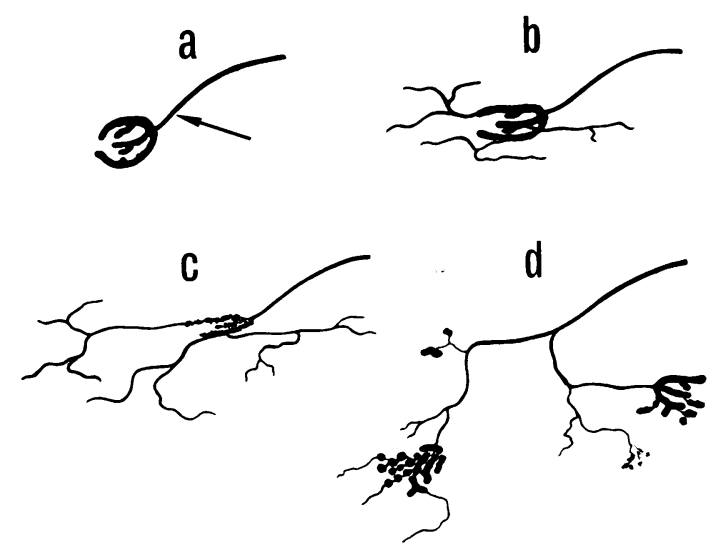

FIG. 15. Diagrammatic representation of the sequence of events induced by botulinum toxin at the motor end-plate. A normal nerve ending (a) shows a single preterminal axon (arrow) innervating the end-plate. Terminal sprouting (b) develops after neuromuscular transmission is blocked by the toxin. The nerve sprouts grow in all directions and branch while the original end-plate may disappear (c). New nerve terminals are formed randomly on some of the nerve sprouts (d). The nerve fibres which were at first ultraterminal sprouts in (b) have now become branched preterminal axons in (d).

and their ramifications across muscle fibres, the eventual cessation of nerve growth, and the reestablishment of functional nerve endings, is much the same in all the muscles affected. The abnormalities in the type and localization of cholinesterase during the period of nerve growth and the eventual abnormalities in the morphology of the subneural apparatuses are also very similar in all the muscles, the principal differences being the rate at which these events occur. Other differences between the muscles include the more severe degree of atrophy of muscle fibres in gastrocnemius than in soleus and the finding of histological abnormalities, such as the variation in diameter of muscle fibres and the persistence of atrophied fibres and of fibres with central nuclei in gastrocnemius for many months. These differences, however, may themselves be a reflection of the much longer period which elapses in gastrocnemius, compared with soleus, between the onset of paralysis and the beginning of motor nerve growth. It is known (Gutmann and Young, 1944) that the re-innervation of muscle becomes progressively more difficult the longer the period of denervation.

In soleus nerve growth commences about one week after the administration of toxin and con- 
tinues until about the fourth or fifth week, after which neuromuscular function is restored as shown by the increase in the diameter of the muscle fibres and the re-appearance of formed subneural apparatuses. In gastrocnemius the nerve growth commences after about three weeks and continues until approximately the sixth or seventh week. The actual period of motor nerve growth is about the same in the different muscles-that is, approximately four weeks. This suggests that the nerve growth itself is an important factor in promoting the recovery of neuromuscular transmission from the blocking effects of botulinum toxin.

There is no evidence in the literature which suggests that botulinum toxin acts preferentially on any one type of cholinergic nerve terminal. The experiments of Dickson and Shevky (1923a, b), Burgen et al. (1949) and Ambache (1949, 1951) all indicate that the toxin blocks transmission at all cholinergic nerve endings in the voluntary and autonomic nervous systems at the same rate. The fact that the muscle fibres of gastrocnemius are paralysed within 24 hours and become atrophied during the weeks before nerve growth commences also indicates that neuromuscular transmission in that muscle is blocked at the same time as in soleus.

The earlier onset of motor nerve growth in soleus and in the deep parts of gastrocnemius cannot be explained as being due to a local concentration of toxin in the depths of the leg muscles for several reasons. One is that the nerve growth in the periphery of gastrocnemius eventually becomes as vigorous and as extensive as in soleus and the atrophy of muscle fibres becomes even more severe peripherally than in the depths of the muscle. Secondly, the differences between the muscles were similar in all the animals studied and no variations were seen which could be correlated with variations in the site of the injection. Another point was that the injection of a dye (pontamine sky blue) in the same volume as that of the toxin did not result in a local concentration of dye in soleus or in gastrocnemius but in a diffusion which stained all the tissues of the leg. Although the exact distribution of toxin in the leg muscles is not known, it seems likely that it would resemble that of the injected dye. There is evidence that the toxin becomes rapidly 'fixed' in the region of the motor end-plates (Burgen, et al., 1949; Zacks, Metzger, Smith, and Blumberg, 1962). It is also well known that in solution the toxin is unstable and is rapidly inactivated by heat and by oxidizing agents. It is thus inconceivable that the delay of several weeks in the appearance of nerve growth at some motor nerve terminals is due to a delay (of weeks) in the toxin molecules reaching those nerve endings. It, therefore, seems $\frac{O}{Z}$ likely that the differences between the various $\mathbb{D}$ muscles are not due to local variations in the distribution of the toxin but are related to intrinsic differences between these muscles.

Soleus in the mouse, as in most mammals, is a 'slow' muscle. Its muscle fibres are uniformly rich $\stackrel{5}{子}$ in succinate dehydrogenase and poor in phosphorylase. The deep muscle fibres of gastrocnemius? show the same enzyme histochemical reactions as $\vec{F}$ those of soleus. These histochemical reactions are $\stackrel{\vec{F}}{+}$ considered (Dubowitz and Pearse, 1964) character- $\frac{}{c}$ istic of 'slow' muscle. The great majority of superficial fibres of gastrocnemius, occupying about $\frac{\omega}{\sigma}$ one-third of the depth of that muscle, give a strong $\varnothing$ histochemical reaction for phosphorylase and a weak o reaction for succinate dehydrogenase and can be $\overrightarrow{0}$ considered to be 'fast' muscle fibres. Very few 'slow' fibres are found in this part of gastrocnemius. $\vec{\omega}$ These observations are in keeping with those of $\mathscr{S}$ Gordon and Holbourn (1949) who noted that in some muscles of the cat the fast fibres lay in the periphery, while slow fibres were found in the depths of the muscle. Many other differences betweero slow and fast muscle fibres are known and have been reviewed by Gutmann (1966).

There is evidence (Buller, Eccles, and Eccle\& 1960; Elul, Miledi, and Stefani, 1968) that the of property of 'fastness' or 'slowness' is conferreg on a muscle fibre by a neural mechanism which $\frac{10}{2}$ distinct from the neuromuscular transmissiof mediated by acetylcholine. Differences in the calibre of motor nerve fibres which innervate fast or slow muscles are also known, the slow muscles being innervated by the smaller nerve fibres. The histological observations recorded in the present paper demonstrate hitherto unknown differences between slow and fast muscle fibres and their innervation. Any explanation of the mechanism of the motor nerve growth induced by botulinum toxin must take these differences into account. It may be that factors similar to those which promote and control motor nerve growth in this experimental situation are also of importance in determining the reactions of muscle fibres and their innervation in naturally-occurring disease processes.

The changes induced by botulinum toxin illustrate the lability of the motor nerve and the muscle which it innervates. The sequence of events which 0 follows the block of transmission demonstrates the structural dependence of the neuromuscular system on its functional integrity. There is much to be learned from the experimental use of neuro- $\sigma$ muscular blocking agents and botulinum toxin, $N$ because of its prolonged action, should be especially $N$ 
useful in determining factors which promote and control nerve growth.

\section{SUMMARY}

A sublethal quantity of botulinum toxin was injected into the muscles of one leg in mice which were killed at intervals ranging from two days to 15 months after the injection. The innervation of the muscles and the distribution of cholinesterase were studied histologically.

The muscle fibres became paralysed and atrophied, and sprouting of nerve fibres from motor nerve terminals was found. Differences between soleus and gastrocnemius muscles were observed. Although both muscles became paralysed within 24 hours, the muscle fibres of soleus (which have the characteristics of slow muscle) became atrophied at a more rapid rate than did those in the superficial region of gastrocnemius (where most fibres have the characteristics of fast muscle). Sprouting of motor nerve fibres from most of the terminals was found in soleus by the sixth to seventh day and progressed for several weeks. The muscle fibres of soleus became atrophied for about three to four weeks but then increased in diameter to within normal limits over the next few weeks. The growth of motor nerves in soleus ceased by about the fourth to fifth week when new end-plates were formed. In the superficial region of gastrocnemius the muscle fibres became progressively atrophied for six weeks or more and became more severely atrophic than those of soleus. Nerve sprouting from the terminals in this part of gastrocnemius was found only after three to four weeks and was maximal by the sixth to eighth week when new end-plates became established and motor nerve growth ceased. Muscle fibres of gastrocnemius then increased in diameter though many remained severely atrophied for many months.

Changes in the localization and characteristics of cholinesterase in both soleus and gastrocnemius were similar, though they occurred at different rates. Cholinesterase activity was found along the motor nerve sprouts. In contrast with normal subneural apparatuses where the principal enzyme component is acetyl (true) cholinesterase, the enzyme associated with the nerve sprouts was mainly non-specific (pseudo) cholinesterase. As new endplates were formed, less pseudocholinesterase was found. In the long surviving animals, persistent ultraterminal nerve fibres, branching of axons, 'blind' nerve endings, and morphologically abnormal subneural apparatuses were found in both muscles.
I should like to thank Professor Peter Daniel and Dr. Sabina Strich for their interest and criticism; Dr. J. Keppie for the botulinum toxin; Dr. B. Lake for advice on histochemical methods; Mr. A. J. Davey and Mr. I. J. Stiff for the microscopic preparations and Mr P. M. Taylor for help with the photography. This work was supported by grants from the Research Fund of The Bethlem Royal and Maudsley Hospitals, the Muscular Dystrophy Group of Great Britain and the Muscular Dystrophy Associations of America, Inc., and formed part of a thesis submitted to the University of the Witwatersrand for the degree of Doctor of Medicine.

\section{REFERENCES}

Ambache, N. (1949). The peripheral action of Cl. Botulinum toxin. J. Physiol. (Lond.), 108, 127-141.

(1951). A further survey of the action of Clostridium Botulinum toxin upon different types of autonomic nerve fibre. Ibid., 113, $1-17$.

Buller, A. J., Eccles, J. C., and Eccles, R. M. (1960). Interactions between motoneurones and muscles in respect of the characteristic speeds of their responses. Ibid., 150, 417-439.

Burgen, A. S. V., Dickens, F., and Zatman, L. J. (1949). The action of botulinum toxin on the neuromuscular junction. Ibid., 109, 10-24.

Coërs, C. E. F., and Woolf, A. L. (1959). The Innervation of Muscle: a Biopsy Study. Blackwell: Oxford.

Couteaux, R. (1958). Morphological and cytochemical observations on the post-synaptic membrane at motor end-plates and ganglionic synapses. Exp. Cell Res. (Suppl), 5, 294-322.

Davis, R., and Koelle, G. B. (1967). Electron microscopic localization of acetylcholinesterase and nonspecific cholinesterase at the neuromuscular junction by the gold-thiocholine and goldthiolacet ic acid methods. J. Cell Biol., 34, 157-171.

Denz, F. A. (1953). On the histochemistry of the myoneural junction. Brit. J. exp. Path., 34, 329-339.

Dickson, E. C., and Shevky, R. (1923a). Botulism. Studies on the manner in which the toxin of Clostridium botulinum acts upon the body. I. The effect upon the autonomic nervous system. J.exp. Med., 37, 711-731.

- and Shevky, E. (1923b). Botulism. Studies on the manner in which the toxin of clostridium botulinum acts upon the body. II. The effect upon the voluntary nervous system. Ibid., 38, 327-346.

Dubowitz, V., and Pearse, A. G. E. (1964). Histochemical aspects of muscle disease, pp. 194-219 in Disorders of Voluntary Muscle. Edited by J. N. Walton. Churchill: London.

Duchen, L. W. (1969). Histological differences between soleus and gastrocnemius muscles in the mouse after the local injection of botulinum toxin. J. Physiol. (Lond.), 204, 17-18P.

innervation of skeletal muscle in the mouse after local injection of Clostridium botulinum toxin. Ibid., 189, 2-4P.

$-1,1968)$. The effects of botulinum toxin on the pattern of innervation of skeletal muscle in the mouse. Quart. J. exp. Physiol., 53, 84-89.

Edds, M. V., Jr. (1950). Collateral regeneration of residual motor axons in partially denervated muscles $J$. exp. Zool., 113, 517-552.

Elul, R., Miledi, R., and Stefani, E. (1968). Neurotrophic control of contracture in slow muscle fibres. Nature (Lond.), 217, 12741275.

Gordon, G., and Holbourn, A. H. S. (1949). The mechanical activity of single motor units in reflex contractions of skeletal muscle. J. Physiol. (Lond.), 110, 26-35.

Gutmann, E. (1966). Basic muscle type differentiation, pp. 132-141 in Exploratory Concepts in Muscular Dystrophy and Related Disorders. Edited by A. T. Milhorat. Excerpta Medica (International Congress Series No. 147): New York.

, and Young, J. Z. (1944). The re-innervation of muscle after various periods of atrophy. J. Anat. (Lond.), 78, 15-43. 
Guyton, A. C., and MacDonald, M. A. (1947). Physiology of botulinus toxin. Arch. Neurol. Psychiat. (Chic.), 57, 578-592.

Henderson, J. R. (1967). The use of silver for intensifying sulfide deposits in the cholinesterase technique. Stain Technol., 42,
101-102.

Hoffman, H. (1950). Local re-innervation in partially denervated muscle: a histophysiological study. Aust. J. exp. Biol. med. Sci., 28, 383-397.

Jirmanova, I., Sobotkova, M., Thesleff, S., and Zelena, J. (1964). Atrophy in skeletal muscles poisoned with botulinum toxin. Physiol. bohemoslov., 13, 467-472.

Koelle, G. B., and Friedenwald, J. S. (1949). A histochemical method for localizing cholinesterase activity. Proc. Soc. exp. Biol. Med., 70, 617-622.
Lehmann, H., Silk, E., and Liddell, J. (1961). Pseudo-cholinesteras Brit. med. Bull., 17, 230-233.

Lewis, P. R. (1961). The effect of varying the conditions in the Koeft technique. Bibl. anat. (Basel), 2, 11-20.

Namba, T., Nakamura, T., and Grob, D. (1967). Staining for ner fiber and cholinesterase activity in fresh frozen sections Amer.J. clin. Path., 47, 74-77.

Pearse, A. G. E. (1960). Histochemistry, Theoretical and Applie@ 2nd Edn. Churchill: London.

Zacks, S. I., Metzger, J. F., Smith, C. W., and Blumberg, J. M. (1962 Localization of ferritin-labelled botulinus toxin in the neurcs muscular junction of the mouse. J. Neuropath. exp. Neurol., 21. 610-633. 